\section{LA-UR- $89-0122 \%$}

Approved for public release; distribution is unlimited.

Title:

Author(s):

WANCHUCK WOO, ZHILI FENG and EDWARD KENIK: ORNL

TAMÁS UNGÁR: Eötvös University, Hungary

BJøRN CLAUSEN: LANSCE-LC, LANL

Intended for:

Proceedings of the 2009 Annual TMS Meeting, San

Francisco, February 15-19, 2009

To be published in Metall. Trans. A

Los Alamos National Laboratory, an affirmative actionequal opportunity employer, is operated by the Los Alamos National Security, LLC for the National Nuclear Security Administration of the U.S. Department of Energy under contract DE-AC52-06NA25396. By acceptance of this article, the publisher recognizes that the U.S. Government retains a nonexclusive, royalty-free license to publish or reproduce the published torm of this contribution, or to allow others to do so, for U.S. Government purposes. Los Alamos National Laboratory requests that the publisher identify this anticle as work performed under the auspices of the U.S. Department of Energy. Los Alamos National Laboratory strongly supports academic freedom and a researcher's right to publish; as an institution, however, the Laboratory does nol endorse the viewpoint of a publication or guarantee its technical correctness. 


\title{
X-ray and Neutron Diffraction Measurements of Dislocation Density and Subgrain Size in a Friction Stir Welded Aluminum Alloy
}

\author{
WANCHUCK WOO ${ }^{\text {a }}$, ZHILI FENG ${ }^{\text {a }}$, TAMÁS UNGÁR ${ }^{b}$, EDWARD KEMIK ${ }^{\text {a }}$, and BJORN CLAUSEN ${ }^{\mathrm{c}}$ \\ "Materials Science and Technology Division, Oak Ridge National Laboratory, Oak Ridge, TN 37831, USA \\ ${ }^{b}$ Department of General Physics, Eötvös University, P.O. Box 32, H-1518, Budapest, Hungary \\ 'Los Alamos Neutron Science Center, Los Alamos National Laboratory, Los Alamos, NM 87545, USA
}

\begin{abstract}
The dislocation density and subgrain size were determined in the base material and friction-stir welding (FSW) of 6061-T6 aluminum alloy. First, the high-resolution x-ray diffraction measurement was performed in the base material. The result of the line profile analysis of the $\mathrm{x}-$ ray diffraction peak shows that the dislocation density is about $4.5 \times 10^{14} \mathrm{~m}^{-2}$ and the subgrain size is about $200 \mathrm{~nm}$. Secondly, the deep penetration capability of the neutrons enables us to measure the diffraction peaks from the mid-plane of the Al plate underneath the tool shoulder at $8 \mathrm{~mm}$ from the tool center during FSW. The broadening peak profiles were analyzed using the Williamson-Hall method. The result shows the dislocation density of about $3.2 \times 10^{15} \mathrm{~m}^{-2}$ and subgrain size of about $160 \mathrm{~nm}$ during FSW. The significant increase of the dislocation density is likely due to the severe plastic deformation during FSW. This study provides an insight to understand the transient behavior of the microstructure.
\end{abstract}

\section{Introduction}

In many thermo-mechanical engineering processes, the severe plastic deformation results in a large amount of dislocations in microstructure [1,2]. It has been reported that the dislocationembedded 'grain structure' is subjected to lattice distortions and strains within grains of materials $[3,4]$. It is the case of friction-stir welding (FSW). FSW is a solid-state joining method utilizing a rotating tool consisting of a threaded pin and tool shoulder to apply the severe plastic deformation and frictional heating into the joining materials [5]. During FSW, the materials softened by heat underneath the rotating tool shoulder flow around the tool pin column with pressure and consolidate behind the tool to form a solid-state continuous region [6]. As a result, the FSW shows a unique grain structure influenced by the severe thermo-mechanical deformation, recovery, and/or recrystallization at elevated temperatures during welding [7-11].

The microstructure of FSW is one of the most important research areas. Although the microstructure of FSW has been extensively studied, very limited quantitative analysis of the grain structure in terms of the dislocation density and subgrain size is available up to date. Detailed information of the grain structure is necessary in FSW because the grain structure (dislocation density and subgrain size) can significantly affect the plastic deformation behaviors such as strain hardening rate and hardening capacity in welds [12]. The grain morphologies and dislocation structures have been observed on various FSW alloys using Transmission Electron Microscope (TEM) and other methods, but these typical observations rely on the "post-mortem" analysis method, which examines the material after processing. Recently, the transient materials behavior "during" processing has been focused to understand the evolution and the final microstructure characteristics.

In-situ observation of microstructure changes has been extremely challenging and many measurement techniques are not applicable. In order to observe the transient microstructure 
changes during FSW, the deep penetration capability of the neutron diffraction is necessary. In the current study, for example, the neutron beam needs to penetrate through about 12-mm thickness of the FSW machine tool and diffract from the FSW Al alloy underneath the machine tool. One major issue in neutron diffraction is the relatively weak neutron fluxes causing difficulties in in-situ experiments to observe the fast, dynamic materials behavior. Thus, in-situ neutron diffraction study of transient materials behavior requires a completely different experimental approach from the conventional study of static problems such as residual stress measurements. For this purpose, we recently developed in-situ time-resolved neutron diffraction method, which drastically improves the temporal resolution of neutron diffraction, enables observation of the transient microstructure changes during FSW [13,14]. In order to achieve the sufficient temporal resolution the quasi-steady state (QSS) was created in the sample by continuous moving of the whole $\mathrm{Al}$ sample plate. In this end, the required temporal resolution (over 10 minuites) of the typical neutron-diffraction measurements can be achieved when utilizing the QSS approach and, thereby, overcome the limitation of the temporal resolution in neutron-diffraction measurements [15].

The diffraction peak profile analysis is a well-established technique for the determination of microstructure in terms of dislocation density and subgrain size in crystalline materials [16-20]. Williamson-Hall peak analysis method suggests that diffraction peak profiles broaden, when subgrains (or crystallites) are small or if the crystal lattice is distorted by lattice defects, especially by dislocations $[3,4]$. The size and strain effects on the diffraction peak broadening can be evaluated separately on the basis of their different $h k l$ dependences $[16,17]$. For example, the Convolutional Multiple Whole Profile (CMWP) fitting procedure has been used to evaluate diffraction peak patterns [18]. In the CMWP, the size and strain profiles are convoluted to produce a theoretically simulated peak pattern. The two defect-related profile functions deconvolute the size and strain profiles for the diffraction peaks from a dislocated crystal [19]. Consequently, the simulated and measured diffraction patterns are compared to each other by a non-linear least squares method and provide the microstructural characteristics. Details are described in elsewhere [16-20].

In this paper, we determined the dislocation density and subgrain size in: (i) the base material (6061-T6 Al alloy) using the high-resolution x-ray diffraction and neutron diffraction, and (ii) the severely deformed zone (stir zone) of FSW 6061-T6 Al alloy using the in-situ time-resolved neutron diffraction. The diffraction peaks measured by x-ray and neutron diffractions were analyzed using the CMWP and Williamson-Hall peak broadening analysis methods. Consequently, the dislocation density and subgrain size were determined "during" FSW and compared to the base material.

\section{Experimental details}

\subsection{Material}

As-received commercial 6061-T6 Al alloy rolled plate was solution-heat treated and aged for 6 hours at $185^{\circ} \mathrm{C}$. The nominal chemical composition in weight percent is $1.0 \mathrm{Mg}, 0.6 \mathrm{Si}, 0.3 \mathrm{Cu}$, and balance Al. Grains of about $200-\mu \mathrm{m}$ size were elongated along the rolling direction with a typical recrystallized rolling texture. The cross-section of the base material and FSW (stir zone) was cold-mounted, polished, and etched with the Keller's reagent for the optical microscopy at room temperature. The grain structure including dislocations was observed from the base material and FSW using TEM. The disc specimen (3 $\mathrm{mm}$ in diameter) was prepared by electrical 
discharge machining (EDM), mechanical grinding to $100 \mu \mathrm{m}$ thickness, and electropolishing using a $30 \%$ nitric acid solution in methanol with 25 voltages for 20 seconds at $-30^{\circ} \mathrm{C}$.

\subsection{X-ray diffraction measurements}

The grain structure of the base material (A1 6061-T6) was measured using X-ray diffraction line profile analysis. The high-resolution micro-beam $x$-ray diffractometer [19] (Nonius FR591) was used with a rotating copper anode $\left(\mathrm{Cu} \mathrm{K} \alpha_{1}\right)$ radiation (wavelength, $\lambda=0.15406 \mathrm{~nm}$ ). The $\mathrm{x}-$ ray beam size is about $0.1 \times 1 \mathrm{~mm}$ on the sample and the scattered radiation is registered by four different position-sensitive imaging detectors located at $300 \sim 600 \mathrm{~mm}$ from the base material. The scattered radiation covered about $2 \theta=30$ to 150 degrees of the angular range of diffractions.

\subsection{FSW and in-situ neutron-diffraction measurements}

We specially designed a FSW machine in real engineering scale. The machine was remotely operated and mounted on the translation stage of Spectrometer for MAterials Research at Temperature and Stress (SMARTS) at Los Alamos Neutron Science Center [21]. The FSW was made on $6.35 \mathrm{~mm}$-thickness 6061-T6 Al alloy plate. The plate sample was continuously traversed at a constant traveling speed of $0.42 \mathrm{~mm} / \mathrm{s}$ as the tool was rotated at 156 revolutions $/ \mathrm{min}$ with a pressure into the sample surface to impose the severe thermo-mechanical deformation on materials. The transverse sides of the plate sample was clamped to constrain the displacement during processing and the clamping was removed after air cooled to $25^{\circ} \mathrm{C}$. In the end, a $760-\mathrm{mm}$ long and $25-\mathrm{mm}$ wide deformation region was made along the middle of the plate width. The hard tool made of an $\mathrm{H}-13$ steel and the diameter of the threaded pin and the tool shoulder was 6.35 and $25.4 \mathrm{~mm}$, respectively. The specimen of the Al plate was $965-\mathrm{mm}$ long (x), 178-mm wide $(\mathrm{y})$, and $6.35-\mathrm{mm}$ thick $(\mathrm{z})$.

We measured diffraction peaks based on the quasi-steady state (QSS) approach described in ref. 22. The QSS is an energy equilibrium state, which is not changing the materials behavior as a function of time. In order to create the QSS, importantly, the whole Al sample plate was continuously traversed along the x-direction, Fig. 1, and simultaneously the neutron diffraction measurements were performed as the deformation zone was being made. The incident neutron beam was focused on a predetermined measurement location $(8 \mathrm{~mm}$ behind the tool center) and the detector collected the diffraction peaks from the defined scattering volume with a scattering vector parallel to the normal direction of the plate using a scattering volume of $12 \mathrm{~mm}^{3}$ defined by the $2(\mathrm{x}) \times 2(\mathrm{y}) \mathrm{mm}^{2}$ square slit of the incident beam and the $3-\mathrm{mm}(\mathrm{z})$ wide radial collimator. The long plate sample dimension (965-mm long) allowed achieving over 20 minutes of the QSS during FSW.

In addition, a neutron diffraction measurement was performed in the base material. The purpose is to obtine the initial state (background) of the neutron diffraction peak profiles from the base material. The peak roadening measured from the base material was compared to that of the "during" FSW. The diffraction measurement of the base material was continued for about 2 hours to obtain the enough statistics in the neutron-diffraction peak intensities from the base material.

\section{Results and discussion}

\subsection{Microstructure analysis}

Figure 2 shows the microstructure of the cross-section of the base material (a commercial 6061-T6 aluminum alloy rolled plate) and FSW 6061-T6 Al alloy. The average grain sizes were 
measured at base material and FSW (stir zone) using optical microscopy. The result shows about 5 , and $120 \mu \mathrm{m}$ in grain size using the linear intercept method, Figs. 2(a) and 2(b). Two TEM bright-field images are also shown in Fig. 2 . The images show the dislocation-embedded grain structure in the base material, Fig. 2(c); and the thermo-mechanically deformed stir zone of FSW, Fig. 2(d). It shows that the as-received rolled Al plate sample contains a large number of dislocations (black line segments) compared to the FSW (stir zone) that is likely to experience the dynamic recrystallization during FSW $[8,9]$.

\subsection{X-ray diffraction peak profile analysis}

Figure 3(a) shows an example of the directly measured $x$-ray diffractogram at the base material (6061-T6 Al alloy). The $\mathrm{x}$-ray diffraction peak profiles were evaluated by the CMWP full pattern fitting procedure. The open circles are the directly measured diffraction peak data and the solid line is a fitted curve using the theoretical diffraction functions obtained by the CMWP fitting procedure $[18,19]$. The indices of each $(h k l)$ reflection are presented for the fcc $\mathrm{Al}$ alloy, and the difference between the measured and fitted pattern is shown in the lower part of the figure. It shows a good fitting result between measured and theoretically simulated profiles. Using the comparison between the measured profiles and theoretically calculated diffraction profiles, the CMWP procedure numerically provides the following independent parameters for the microstructure characterization in the current base material [18]: (i) the median ( $m, 110 \pm 10$ $\mathrm{nm})$ and (ii) the variance $(\sigma, 0.51 \pm 0.05)$ of the log-normal size distribution function, (iii) the mean subgrain size $\langle\mathrm{x}\rangle_{\text {area, }}$, and (iv) the dislocation density $(\rho)$. Note that the area-weighted mean subgrain size can be calculated using the following equation [19]:

$$
<x>_{\text {area }}=m \exp \left(2.5 \sigma^{2}\right)
$$

Using the CMWP procedure, the numerically calculated dislocation densities and average subgrain size is about $4.5 \pm 0.5 \times 10^{14} \mathrm{~m}^{-2}$ and $200 \pm 20 \mathrm{~nm}$, respectively, in the base material.

Williamson-Hall plot shows the qualitative behavior of diffraction peak broadening using the full width at half maximum (FWHM) as a function of $K$, where $K=2 \sin \theta / \lambda, \theta$ and $\lambda$ are the diffraction angle and the wavelength of the x-ray, respectively [16]. The FWHM are plotted as $\Delta K=2 \cos \theta(\Delta \theta) / \lambda$ in $1 / \mathrm{nm}$ scale, where $\Delta \theta$ is the FWHM obtained from each $(h k l)$ peak in Fig. 3(a). Note that the $C$ is the dislocation contrast factor, which is determined by the elastic anisotropy and the dislocation type of materials (details in section 3.3 and ref. 17). Consequently, Fig. 3(b) shows the Williamson-Hall plot of the base material and presents the line broadening $(\Delta K)$ behavior among the different $(h k l)$ peaks.

\subsection{Neutron diffraction peak profile analysis}

In order to study the transient materials behavior, the neutron diffraction measurements were performed underneath tool shoulder during FSW. Figure. 4(a) show the neutron diffraction peaks measured in the base material and during FSW. The measured diffraction peaks in the raw data were analyzed using the single peak fitting method, which refines each $(h k l)$ reflection of the measured diffraction peaks in the General Structure Analysis System (GSAS) [23]. It determines the interplanar spacing ( $\mathrm{d}$-spacing), peak intensity, and peak broadening among the differnet $(h k l)$ grains.

The Williamson-Hall peak broadening analysis suggests that broadening of the diffraction peak profile $\left(\Delta K, \mathrm{~nm}^{-1}\right)$ can be written as a combination of the grain size $(0.9 / D)$ and strain $\left(\Delta K^{D}\right)$ 
effects $[3,16]: \Delta K=0.9 / D+\Delta K^{D}$ where $K=1 / d, \Delta K=-K(\Delta d / d)$, and $D$ is the average subgrain (crystallite) size. The $d(\mathrm{~nm})$ is the d-spacing and $\Delta d(\mathrm{~nm})$ is the FWHM obtained from the single peak fitting of the diffraction data. The Williamson-Hall plot has been modified as replacing $K$ by $K^{2} \mathrm{C}$ based on the following equation $[16,17]$ :

$$
(\Delta K)^{2}=(0.9 / D)^{2}+\left(\pi A^{2} b^{2} / 2\right) \rho\left(K^{2} C\right)+O\left(K^{2} C\right)^{2}
$$

where $A$ is a constant depending on the effective outer cutoff radius of dislocations, $b$ is the Burgers vector of dislocation $(0.286 \mathrm{~nm}$ in $\mathrm{Al}), \rho$ is the dislocation density, and $O$ indicates noninterpreted higher-order terms. Strain anisotropy among the each $(h k l)$ reflection has been rationalized by the dislocation contrast factor $(C)$ in the modified Williamson-Hall plot, Fig. 4(b).

The dislocation contrast factor $\left(C_{h k l}\right)$ become a linear function of the $(h k l)$ invariant of the reflections. In the polycrystalline fcc Al metal: $C_{h k l}=0.2\left[1-q\left(h^{2} k^{2}+h^{2} l^{2}+k^{2} l^{2}\right) /\left(h^{2}+k^{2}+l^{2}\right)^{2}\right]$, where the 0.2 is the average dislocation contrast factor determined by the elastic anisotropy factor, dislocation type, and single crystal elastic constants (see the Fig. 1 in ref. 17). Hence, the $C_{h k l}$ is presented as a function of $q$ parameter in each $(h k l)$ reflection. Put the $C_{h k l}$ into the Eq. (2) yields the theoretical calculation of the $\Delta K$. The theoretical calculated $\Delta K$ and each constant coefficient including parameter $q$ can be determined when the difference between the calculated and measured $\Delta K$ is the minimum using the least-square fitting method [19]. As a result, the $\Delta K$ was modified and fitted among the $(h k l)$ reflections as a function of $K^{2} C$ as shown in Fig. $4(\mathrm{~b})$.

\subsection{Dislocation density and subgrain size}

The modified Williamson-Hall plot, Fig. 4(b), provides two important microstructural features $[16,17]$. First, the slope of the linear regression is proportional to the microstrain. The slope corresponding to the "during" FSW is considerably higher than that of the base material. It indicates much larger microstrains mainly caused by dislocations during FSW. Second, the intercept of the linear regression through the data points at $K=0$ is a rough size estimation of the coherent scattering domains such as subgrain size of microstructure. Accordingly, the fitting curves provide the intercept constant, $(0.9 / D)^{2}$, and the slope of the fitting curve, $\left(\pi A^{2} b^{2} / 2\right) \rho$, in the base material and FSW, Table 1.

In order to calculate the dislocation density, it is necessary to determine the $A$ value of the second coefficient $\left(\pi A^{2} b^{2} \rho / 2\right)$ in Eq. (2). Presumably the dislocation density of the base material is identical when measured using the neutron or $\mathrm{x}$-ray diffraction methods. Thus, the dislocation density $\left(\rho=4.5 \pm 0.5 \times 10^{14} \mathrm{~m}^{-2}\right)$ obtained from the $\mathrm{x}$-ray diffraction puts an equal to the second coefficient with $b=0.286 \mathrm{~nm}$ in Al. It determines the unknown constant $A$ as 0.63 . Note that this value was applied for the calculation of the dislocation density "during" FSW.

Figure 5 shows the analyzed dislocation density and subgrain size in the base material and FSW 6061-T6 Al alloy using neutron diffraction. The modified Williamson-Hall analysis results were summarized in Table 1 . The dislocation density is considered to be closely related to the straining due to the severe plastic deformation and the recrystallization process in FSW [7-9]. Compared to the initial dislocation density $\left(4.5 \times 10^{14} \mathrm{~m}^{-2}\right)$, which has been found in the typical as-received rolled Al plate [24], significant increase of the dislocation density is obvious up to $3.2 \times 10^{15} \mathrm{~m}^{-2}$ during FSW. It is likely that the measurement location (underneath the tool shoulder at $8 \mathrm{~mm}$ from the tool centerline) experiences a severe straining and deformation with high strain rates. Meanwhile, there is no significant change in the subgrain size $(160 \mathrm{~nm})$ during FSW compared to the base material $(200 \mathrm{~nm})$. It has been reported that the crystallite (particle) 
size obtained using diffraction methods can be equivalent to the mean size of domains such as sub-grains or dislocation cells due to the coherently scattered $x$-rays or neutrons from such domains [4]. Consistently, it is clear that the subgrain size (nm scale) measured by the neutron diffraction, Table 1, is much smaller than the grain size ( $\mu \mathrm{m}$ scale) observed in Fig. 2.

The dislocation density of the grain structure can significantly influence on the strain hardening behavior associated with dislocation movements during tensile deformation in FSW [20]. Therefore, this in-situ observation of transient microstructure is important to understand the complicate FSW process, which includes the severe plastic deformation, subdivision of grains, and dynamic recrystallization at elevated temperature.

\section{Conclusions}

The dislocation density and subgrain size were determined in the base material and FSW 6061-T6 Al alloy. In-situ time-resolved neutron diffraction measurements were performed during FSW. The diffraction peak broadening analysis using the modified Williamson-Hall plot quantifies the dislocation density of about $3.2 \times 10^{15} \mathrm{~m}^{-2}$ and the average subgrain size of about $160 \mathrm{~nm}$. The dislocation density during FSW is 7 times higher than the initial dislocation density $\left(4.5 \times 10^{14} \mathrm{~m}^{-2}\right)$ of the base material, which was measured using the high-resolution $\mathrm{x}$-ray diffraction. The significant increase of the dislocation density during FSW is most likely due to the severe plastic deformation imposed by the stirring tool pin. This study shows the fast, transient grain structure inside a bulk Al alloy during FSW with the help of high-resolution $\mathrm{x}$-ray diffraction and in-situ time-resolved neutron diffraction measurement methods.

\section{Acknowledgements}

This research is sponsored by the Laboratory Directed Research and Development program of Oak Ridge National Laboratory, managed by UTBattelle, LLC for the US Department of Energy under Contract no. DE-AC05-00OR22725. This work is also supported by the NSF International Materials Institutes Program under contract DMR-0231320. TU is grateful to the Hungarian National Science Foundation OTKA no. 71594 and no. 67692. Research at the Oak Ridge National Laboratory SHaRE User Center supported by the Division of Materials Sciences and Engineering, Office of Basic Energy Sciences, U.S. Department of Energy, under Contract No. DE-AC05-00OR22725 with UT-Battelle, LLC. Authors would like to thank H. Choo, L. Balogh, T. A. Sisneros, P. K. Liaw, and D. W. Brown for their help.

\section{References}

[1] Y. Wang, M. Chen, F. Zhou, and E. Ma: Nature, 2002, vol. 419, 912-15.

[2] R. Valiev: Nature Mater., 2004, vol. 3, 511-16.

[3] M.A. Krivoglaz: Theory of X-ray and Thermal Neutron Scattering by Real Crystals, Plenum Press, New York, 1996.

[4] B.D. Cullity, and S.R. Stock: Ements of X-ray Diffraction, Prentice Hall, Upper Saddle River, 2001.

[5] M.W. Mahoney, C.G. Rhodes, J.G. Flintoff, R.A. Spurling, and W.H. Bingle: Mater. Trans. A, 1998, vol. 29, 1955-64.

[6] R.S. Mishra and Z.Y. MA: Mater. Sci. Eng. R, 2005, vol. 50, 1-78.

[7] L.E. Murr, G. Liu, and J.C. Mcclure : J. Mater. Sci., 1998, vol. 33, 1243-51.

[8] K.V. Jata and S.L. Semiatin: Scripta Mater, 2000, vol. 43, 743-49.

[9] J.Q. Su, T.W. Nelson, R. Mishra, and M. Mahoney: Acta Mater., 2003, vol. 53, 713-29. 
[10] P.B. Prangnell, and C.P. Heason: Acta Mater., 2005, vol. 53, 3179-92.

[11] W. Woo, H. Choo, D.W. Brown, and Z. Feng: Metal. Mater. Trans. A, 2007, vol. 38, 69-76.

[12] A. Simar, Y. Bréchet, B. de Meester, A. Denquin, T. Pardoen: Acta Mater., 2007, vol. 55, 6133-43.

[13] W. Woo, Z. Feng, X-L. Wang, K. An, H. Choo, C.R. Hubbard, and S.A. David : Appl. Phys. Lett., 2006, vol. 88, 248623.

[14] W. Woo, Z. Feng, X-L. Wang, D.W. Brown, B. Clausen, K. An, H. Choo, C.R. Hubbard, and S.A. David: Sci. Tech. Welding Joining, 2007, vol. 12, 298-303.

[15] W. Woo, Z. Feng, X-L. Wang, K. An, W.B. Bailey, S.A. David, C.R. Hubbard, and H. Choo: Residual stresses VII., 2006, vol. 524-525, 387-92.

[16] T. Ungár and A. Borbély: Appl. Phys. Lett., 1996, vol. 69, 3173-75.

[17] T. Ungár, I. Dragomir, Á. Révész, and A. Borbély: J. Appl. Cryst., 1999, vol. 32, 992-1002.

[18] G. Ribárik, T. Ungár, and J. Gubicza: J. Appl. Cryst., 2001, vol. 34, 669-76.

[19] T. Ungár, J. Gubicza, G. Ribárik, and A. Borbély: J. Appl. Cryst., 2001, vol. 34, 298-310.

[20] W. Woo, L. Balogh, T. Ungár, H. Choo, and Z. Feng : Mater. Sci. Eng. A, 2008, vol. 498, 308-13 (2008).

[21] M.A.M. Bourke, D.C. Dunand, and E. Ustundag : Appl. Phys. A, 2002, vol. 74, S1707-09.

[22] K. Masubuchi: Analysis of Welded Structures, Pergamon, New York, 1980.

[23] A.C. Larson and R.B. Von Dreele: General Structure Analysis System (GSAS), Rep. LAUR 86-748, Los Alamos National Laboratory, Los Alamos, 2004.

[24] J. Gubicza, N.Q. Chinh, Z. Horita, and T.G. Langdon: Mater. Sci. Eng. A, 2004, vol. 387, 55-59. 


\section{TABLE 1}

\begin{tabular}{ccc}
\hline & Base material & During FSW \\
\hline$(0.9 / \mathrm{d})^{2}$ & $2.0 \times 10^{-5}$ & $3.6 \times 10^{-5}$ \\
$\left(\pi \mathrm{A}^{2} \mathrm{~b}^{2} / 2\right) \rho$ & $3.6 \times 10^{-5}$ & $2.6 \times 10^{-4}$ \\
\hline Subgrain size $(\mathrm{nm})$ & 200 & 160 \\
Dislocation density $\left(\mathrm{m}^{-2}\right)$ & $4.5 \times 10^{14}$ & $3.2 \times 10^{15}$ \\
\hline
\end{tabular}

Table. 1. Summarize of the coefficient of the fitting curve in the modified Williamson-Hall plot, Fig. 4(b), in the base material and FSW. The intercept of the linear regression and the slope of the linear regression in the fitting curve are equivalent to the $(0.9 / D)^{2}$ and $\left(\pi A^{2} b^{2} / 2\right) \rho$ terms in Eq. (2), respectively. It determines the subgrain size and dislocation density in the base material and "during" FSW of Al 6061-T6 alloy. 


\section{FIGURE 1}

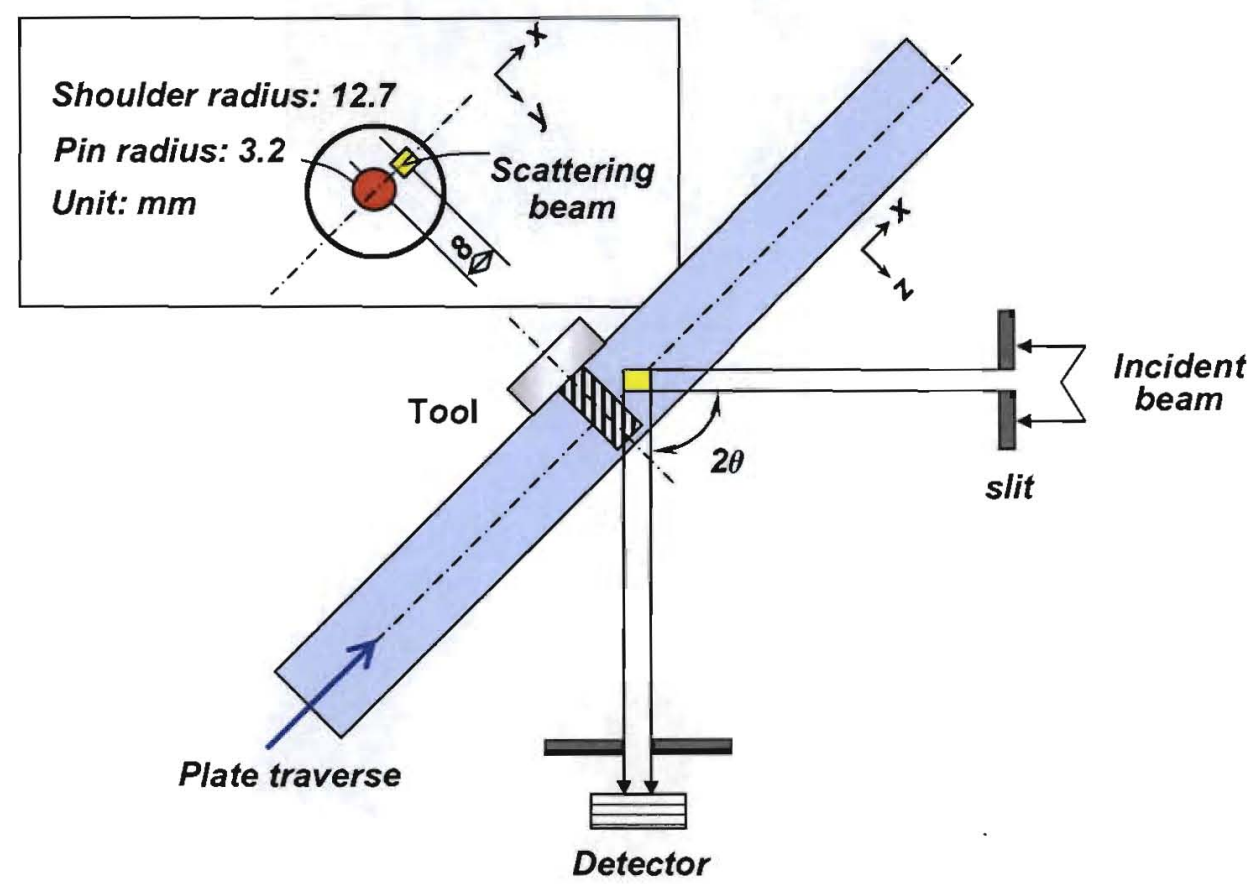

Fig. 1. Experimental setup of the in-situ time-resolved neutron diffraction measurement during FSW. The FSW machine was mounted on the neutron diffractometer and real time measurements were performed as the rotating FSW tool imposes the severe thermo-mechanical deformation on sample. The inset presents the location of the neutron beam, which was predetermined at $8 \mathrm{~mm}$ behind the tool center underneath the tool shoulder. 


\section{FIGURE 2}
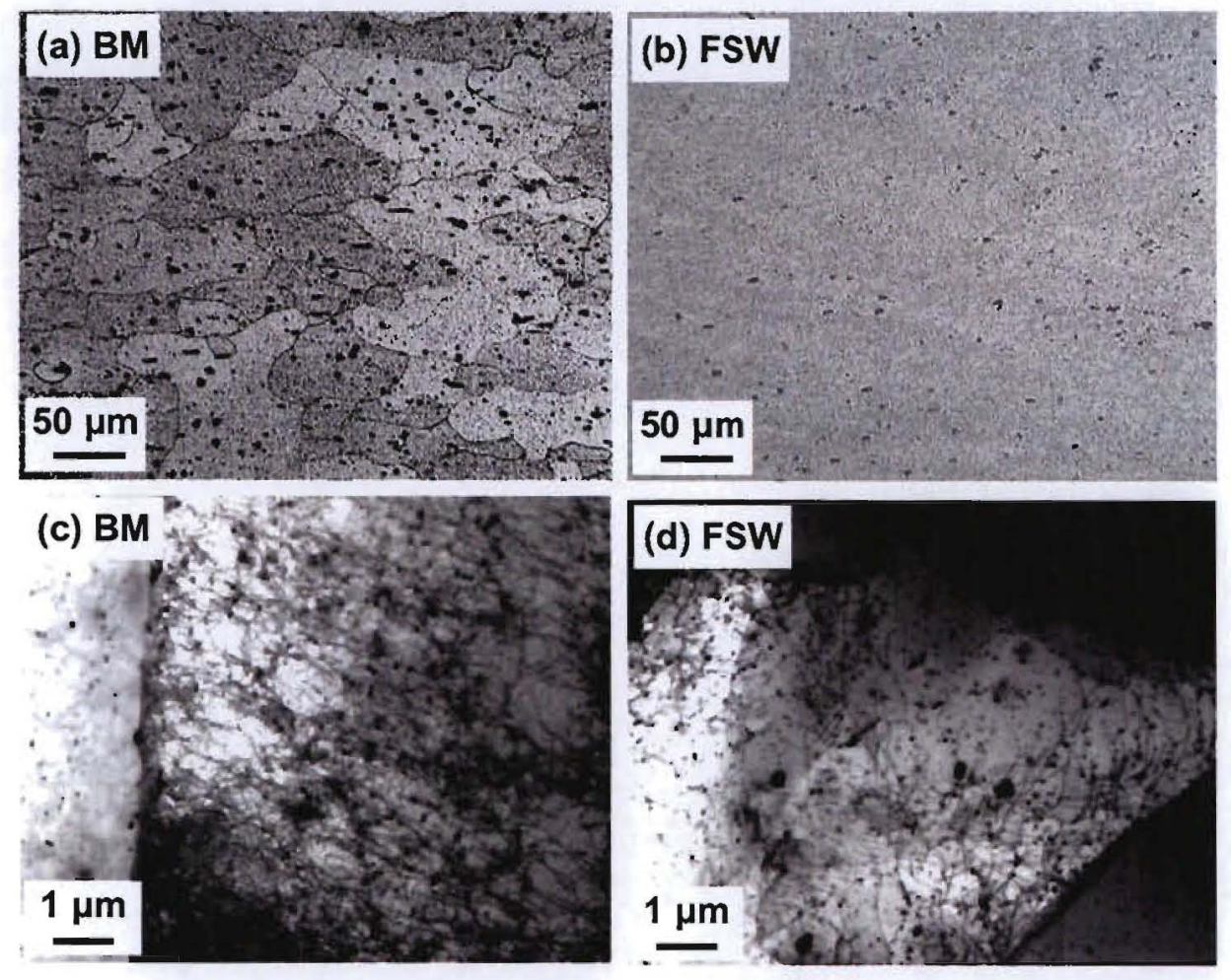

Fig. 2. Microscopy of the 6061-T6 Al alloy: (a) base material and (b) FSW (stir zone). TEM bright-field images of: (c) base material and (d) FSW. The black line segments and dots indicate dislocations and precipitates in the grain structure of TEM, respectively. 
FIGURE 3

(a)
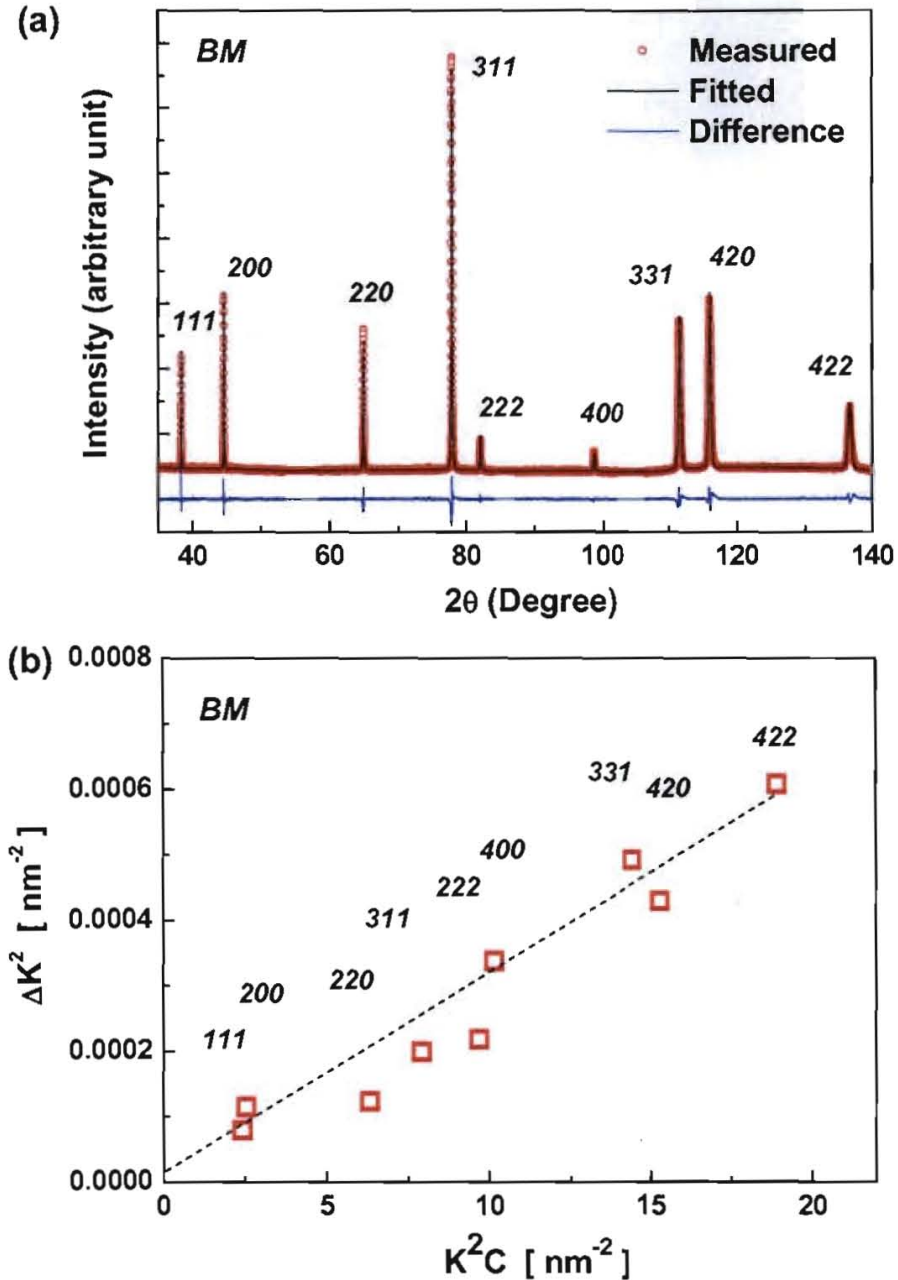

Fig. 3. (a) The x-ray diffraction measured (open circles) and CMWP procedure fitted (solid line) patterns among each $(h k l)$ in the base material of Al 6061-T6 alloy. (b) Peak broadening analysis using the modified Williamson-Hall plot. The FWHM (full width at half maximum, $\Delta K$ ) in each $(h k l)$ peak is presented as a function of $K^{2} C$ ( $K$ is defined as $2 \sin \theta / \lambda$, where $\theta$ and $\lambda$ are the $\mathrm{x}$-ray diffraction angle and the wavelength, respectively, and $C$ is the dislocation contrast factor). 


\section{FIGURE 4}
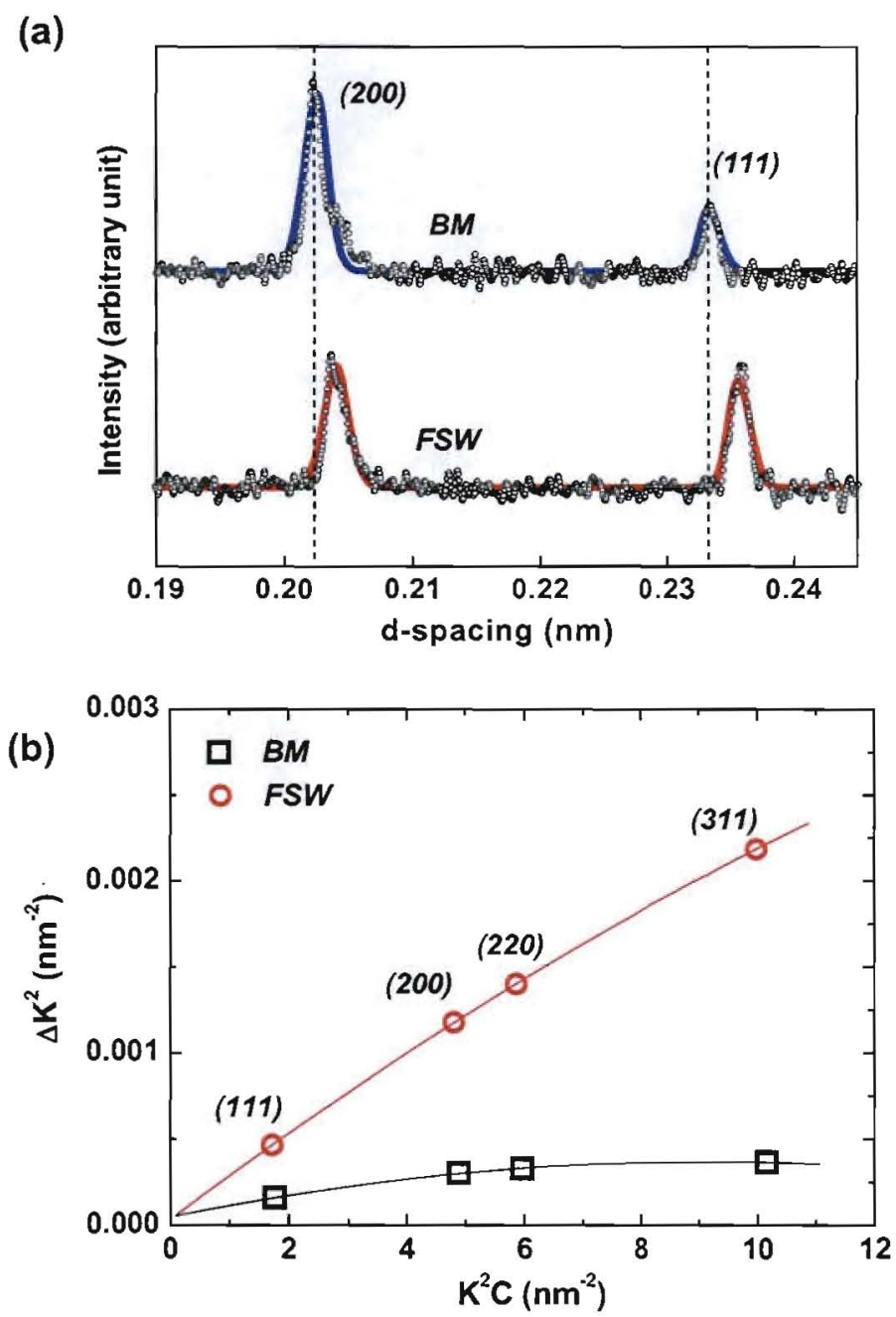

Fig. 4. (a) Neutron diffraction patterns in the (111) and (200) reflections. The gray dots indicate the directly measured neutron diffraction results and the overlapped profiles are Rietveld peak refinements using GSAS. The neutron diffraction patters were measured from the base material (BM) and "during" FSW. (b) Peak broadening analysis using the modified Williamson-Hall plot. The $K$ is defined as $I / d$ ( $d$ is the d-spacing in each hkl peak), $\Delta K$ is driven as $-K(\Delta d / d)$ ( $\Delta d$ is the FWHM obtained in neutron diffraction peak), and $C$ is the dislocation contrast factor. 


\section{FIGURE 5}

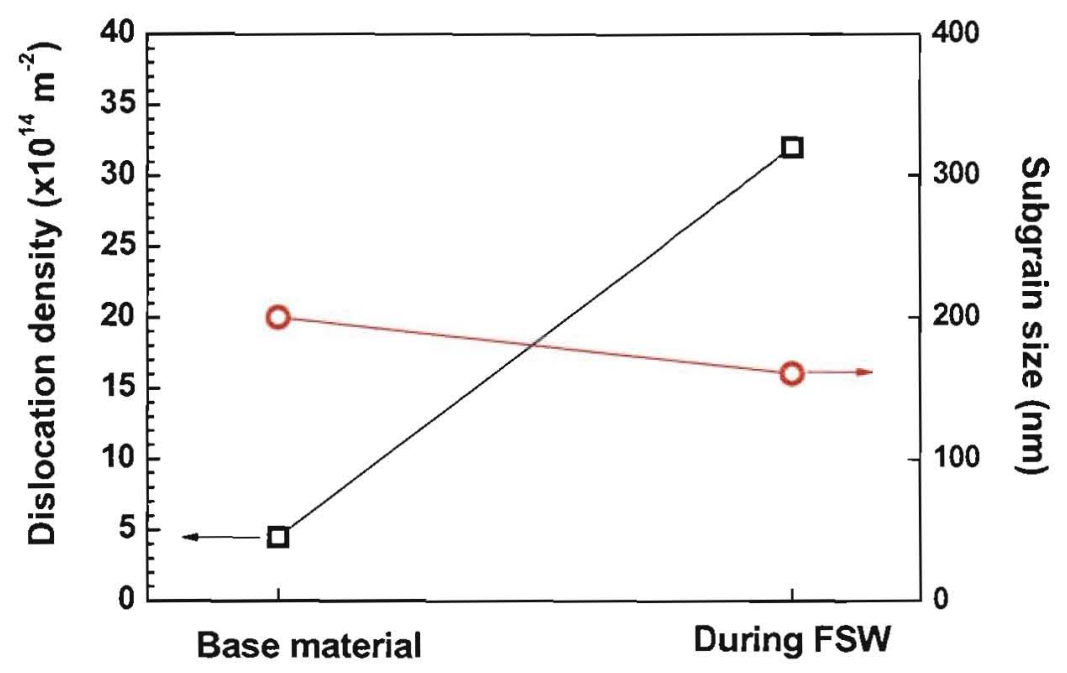

Fig. 5. Dislocation density and subgrain size in the base material and "during" FSW of Al 6061T6 alloy. The interception of the fitted curve, Fig. 4(b), provides a subgrain size (when $\mathrm{K}=0$ in Eq. 2) and the slope of the fitting curve determines the dislocation density. 